\title{
Synergistic integration of computational fluid dynamics and experimental fluid dynamics for ground effect aerodynamics studies
}

T J Barber*, G Doig, C Beves, I Watson, and S Diasinos

School of Mechanical and Manufacturing Engineering, University of New South Wales, Sydney, Australia

\begin{abstract}
This article highlights the 'synergistic' use of experimental fluid dynamics (EFD) and computational fluid dynamics (CFD), where the two sets of simulations are performed concurrently and by the same researcher. In particular, examples from the area of ground effect aerodynamics are discussed, where the major facility used was also designed through a combination of CFD and EFD. Three examples are than outlined, to demonstrate the insight that can be obtained from the integration of CFD and EFD studies. The case studies are the study of dimple flow (to enhance aerodynamic performance), the analysis of a Formula-style front wing and wheel, and the study of compressible flow ground effect aerodynamics. In many instances, CFD has been used to not only provide complementary information to an experimental study, but to design the experiments. Laser-based, non-intrusive experimental techniques were used to provide an excellent complement to CFD. The large datasets found from both experimental and numerical simulations have required a new methodology to correlate the information; a new post-processing method has been developed, making use of the kriging and co-kriging estimators, to develop correlations between the often disparate data types.
\end{abstract}

\section{INTRODUCTION}

\subsection{Ground effect aerodynamics and its simulation}

In the field of aerodynamics, computational fluid dynamics (CFD) and experimental fluid dynamics (EFD) are both widely used. However, it is common for the studies to be conducted separately, by different groups of researchers, with a comparison made between the two datasets at the end of the project. It can often be difficult to numerically replicate the experimental conditions, as important (to the CFD practitioner) information may not be available. Error analysis may be troublesome to compare and sometimes necessary simplification of the experiment may make the CFD a more realistic model, but no longer a useful one for comparison with the experimental data. A distinction can be made between 'serial' studies, where the experiments are first performed and the computational studies follow, and 'parallel' studies, where the computations and experiments are conducted at the same time, but not in a collaborative fashion; the ultimate goal would be for computations and experiments to be conducted in a 'synergistic' fashion, where each approach informs the other, and where the final product of the research is improved because of the interaction between the two [1]. In this article, examples of synergistic studies, utilizing CFD and EFD for the study of ground effect aerodynamics, are described. In each case, the computational and experimental studies are performed by the same researcher, with each component of the study informing the other (Fig. 1).

Ground effect aerodynamics has been an active area of research at UNSW for the past 10 years. Our group has considered a range of topics relevant to this 


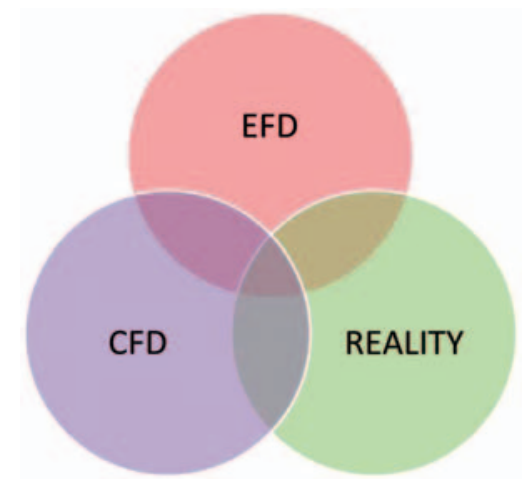

Fig. 1 Synergistic integration of CFD and EFD

interesting field of aerodynamics, and in all cases, the use of CFD and EFD have been used extensively, and in a completely integrated manner. Often this has been from a desire to consider a full-scale, more realistic version of the problem being considered; there is no facility to study a full-size Formula One car, but good measurements for a much smaller experimental case can be made, and CFD can be conducted for either case. By integration of the results and design of the overall methodology for gaining the flow field information, it is possible to get closer to the real world case than by through the separate use of either CFD or EFD. The researchers in our group are not exclusively CFD or EFD practitioners, but expected to be proficient in both, and the integration of the two techniques has also been an active area of research for the group [2-4]. It is only by using both processes that the best integration can be gained, as, for example, experiments will be carefully designed with the CFD model in mind. Experiments may be modified from their original proposal, purely to allow a better constrained CFD boundary condition to be set.

For small ground clearances, like those for automotive purposes, the only accurate experimental way of conducting research into ground effect requires the use of a moving ground facility. CFD cases of the possible alternative ground plane representation techniques demonstrate that the flow field around the aerofoil is substantially changed when using the alternative methods [5].

Ground effect aerodynamics has mostly been studied in a subsonic context [6, 7], but there are also transonic and supersonic applications in which the proximity of the ground is an important parameter, such as land speed record cars, low-flying military aircraft, or projectiles close to the ground or solid obstacles. In order to conduct experiments to study the aerodynamics of supersonic objects in ground effect, particular consideration must be given to the way in which the ground is represented. In a wind tunnel environment with a fixed model, ideally a moving ground would be used for the greatest physical realism [6, 7], but this is impractical at supersonic speeds. It is arguably more straightforward to move the object through quiescent air using, for example, a rocket-sled testing facility [8] or a ballistic range [9], but in addition to the enhanced complexity of all diagnostics related to free-flight measurements, these facilities are generally expensive and may also be subject to restricted military access.

By contrast, supersonic blowdown tunnels are relatively common and accessible, and here a study is detailed to investigate the applicability of the most suitable subsonic ground effect modeling techniques for supersonic applications in such facilities. The extension to transonic conditions is then examined. The numerical modelling was an integral part of this process as the creation of a moving ground in CFD is a trivial task (Fig. 2). The two less ideal experimental options were then compared to the best-case scenario.

In the case of the transonic experiments, CFD was used in the initial stages of the design of the experiments, first to determine conditions at which there was likely to be early onset of shock waves due to the ground effect, and second to tune the design of the sting and endplates to produce minimal interference to the flow on the wing(s). In the course of this numerical programme, it was determined that the flow would still be quite heavily influenced by the tunnel walls and endplates. Two-dimensional (2D)

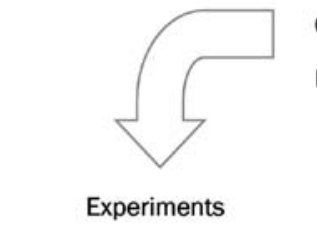

Assessment of apparatus design and performance

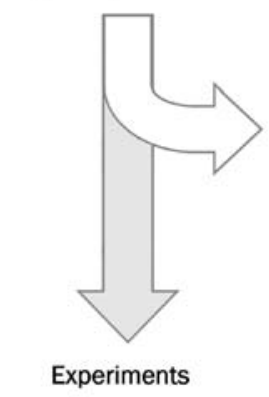

Comprehensive smallscale, low-Re results
CFD of proposed model

Design of apparatus

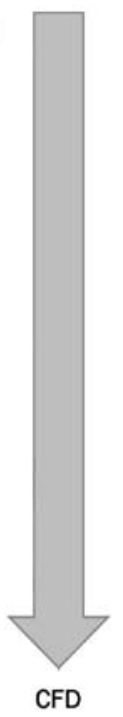

Comprehensive fullscale, high-Re results

Fig. 2 Role of transonic experiments in cooperation with CFD 
CFD conducted during the experimental programme confirmed that a full three-dimensional (3D) representation of the apparatus was required. In validating the high-fidelity numerical results against the data from the wind tunnel, additional features of the flowfield which could not be determined from the experiments alone were revealed, such as transient shock movement and boundary layer behaviour.

\subsection{Overview of experimental and computational approaches}

The $235 \times 340 \mathrm{~mm}^{2}$ UNSW moving ground wind tunnel used for the subsonic experimental measurements is shown in Fig. 3. The inlet has turbulence damping screens positioned between the inlet fairing and the 7.8:1 contraction ratio section and a 5-Hp fan is used to draw the flow through the tunnel. Test section flow angularity is less than $0.2^{\circ}$ and the laser doppler anemometry (LDA) measured free stream turbulence intensity is 1 per cent with a maximum test section speed of $15 \mathrm{~m} / \mathrm{s}$.

As this facility was developed for projects that would use a strong CFD component, the use of measurement techniques which were non-intrusive and also produced large amounts of field data (for better comparison with numerical results) was a high priority. Three main systems are used for measurement in the tunnel.

1. Qualitative flow field using laser sheet flow visualization, with a range of $\mathrm{He}-\mathrm{Ne}$ and $\mathrm{Nd}-\mathrm{YAG}$ lasers, smoke for seeding and digital camera recording.

2. Quantitative analysis using an in-house particle imaging velocimetry (PIV) system. The PIV system uses an EKSPLA NL301-2G unit to generate the primary laser for the PIV system and comprises two lasers (each containing an Nd:YAG rod, flashlamp, and Pockels Cell) and a dichroic mirror to separate the green wavelength $(532 \mathrm{~nm})$ from the (a)

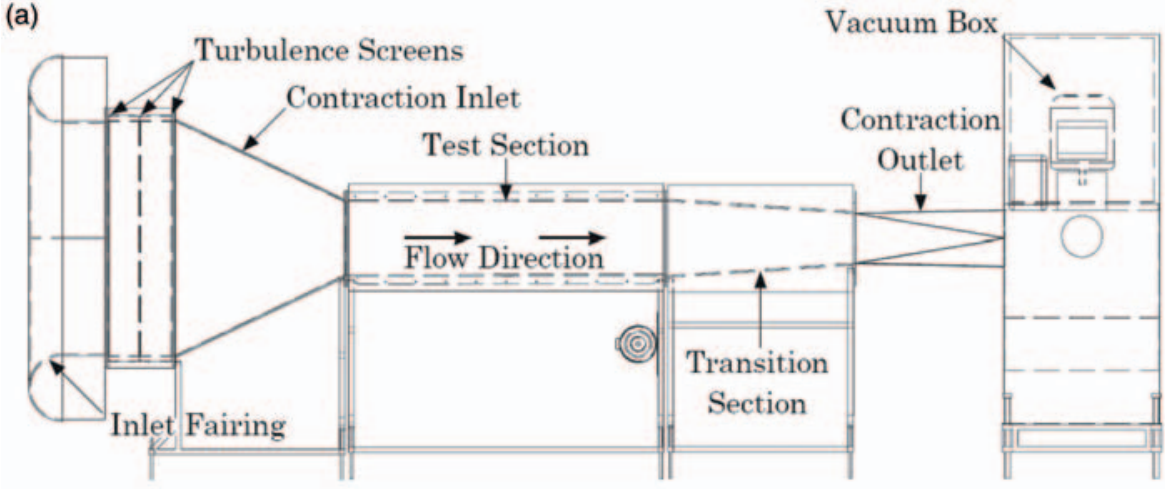

(b)

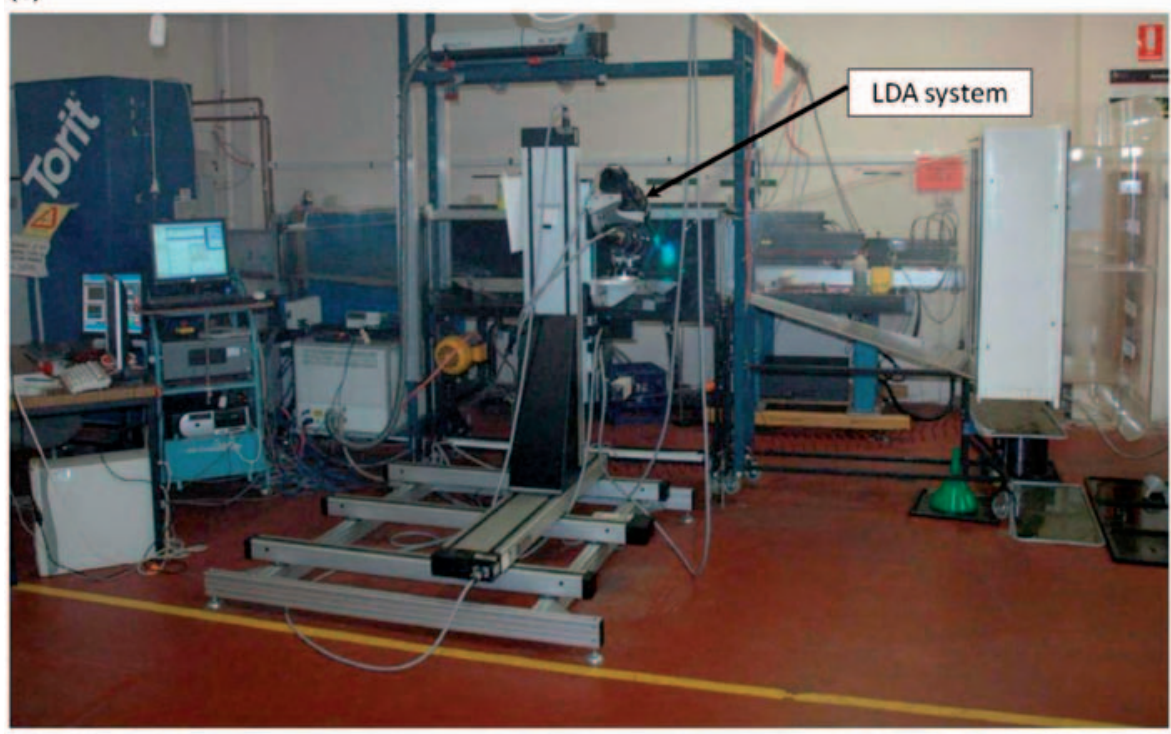

wind tunnel with LDA in use

Fig. $3225 \times 340 \mathrm{~mm}^{2}$ UNSW wind tunnel 
infrared (1064 nm). Using the commercially available image analysis software VidPIVv4.6 from ILA $\mathrm{GmbH}$, processing of the captured images is possible.

3. Quantitative analysis using a Dantec 3D LDA system. A Coherent INNOVA 70C 5W Argon Ion (Arg-Ion) laser supplies the primary beam to the transmitter box, then to the LDA probe heads. The $85-\mathrm{mm}$ probe heads operate in a 3D, coincident, backscatter mode and are mounted to a lightweight three-axis traverse system. High data rates were achieved by a special technique of alignment using a CCD camera allowing beam alignment to a resolution of 5 pixels.

The tunnel walls (and many model components) were manufactured from high quality perspex to allow laser flow measurement techniques to be used anywhere in the tunnel. Atomized vegetable oil seeds the flow for LDA or PIV with particle diameter of $1 \mu \mathrm{m}$, giving a typical particle slip velocity of less than 1 per cent.

Before construction of the wind tunnel, a number of CFD models were developed to ensure the tunnel delivered the flowfield required. A 3D model of the tunnel in the room was used to determine the optimum location and placement relative to walls [10]. A detailed 2D model was used to consider different options for the moving ground placement within the tunnel, and options for leading edge boundary layer removal. Initial design for the most optimal flow consistency and also minimal turbulence (as it was expected the moving ground itself would introduce turbulent energy into the flow) throughout the test section was conducted using CFD. The numerical analyses were carried out using 2D, finite volume Reynolds-averaged Navier-Stokes (RANS) simulations with the $k-\epsilon$ RNG turbulence model, with 350000 grid points and wall $y^{+}=2^{*}$; allowing enhanced wall treatment with pressure gradient effects. The target operational free stream velocity was $10 \mathrm{~m} / \mathrm{s}$ in the $340 \times 225 \mathrm{~mm}^{2}$ cross-section test section $\left(R e_{H}=\leftarrow\right.$ $\left.2.8 \times 10^{5}\right)$. The contraction inlet and test section expansions were designed by numerical analysis and based on similar facilities in the literature $[\mathbf{1 1}, \mathbf{1 2}]$. When the design was considered to be optimized, the tunnel and moving ground were constructed inhouse, and measurements taken with the LDA system to validate the flow-field in the test section. The LDA measurements were taken using a $2 \mathrm{D}$ probe rotated down towards the belt so that measurements could be taken close to the belt surface. Velocity profiles were taken in various positions throughout the test section;

\footnotetext{
${ }^{*} y^{+}$is a non-dimensional wall distance for a wall-bounded flow.
}

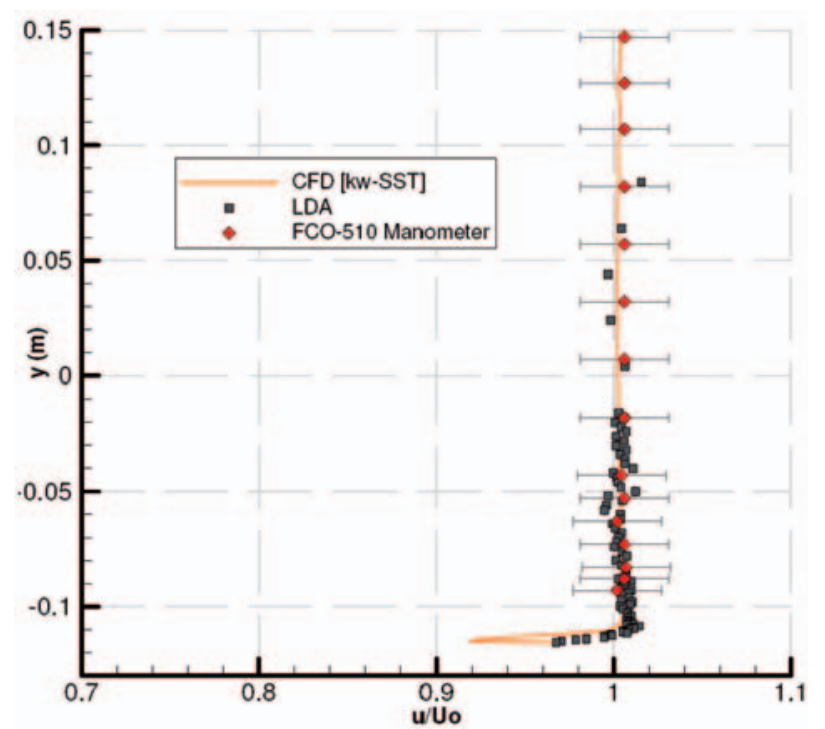

Fig. 4 Velocity profile in test section, and comparison with CFD results

a typical comparison with CFD is shown in Fig. 4, comparing numerical predictions with 2D LDA and Pitot-static measurements (where $u$ is the local velocity and Uo the freestream velocity). The boundary layer was not completely removed from the ground, as was expected from the CFD model; however, the height of the boundary layer and the velocity variation is negligible.

CFD simulations in our group are generally conducted using the Fluent finite volume RANS solver (some research is carried out using LES but this is not detailed in this article). Meshes are generally created using the mesh generator, Gambit. A strong emphasis on validation and verification is given, following the recommendations of Roache [13] and the AIAA [14]. Cases are run in either our local CFD laboratory, where machines run Fluent under Linux, or using either our in-house cluster or the remotely located ac3 (ac3.edu.au) cluster, an SGI Altix 4700 64-bit shared memory machine, with 128 Dual-Core 1.6-GHz CPUs and 1 TB RAM.

For comparisons with experiments in the moving ground wind tunnel, accurate boundary conditions were possible by directly measuring the velocity distribution and turbulence intensity for the relevant test with the 3D LDA system. Full CAD data existed for the wind tunnel and test models; thus, dimensional accuracy of the numerical model was assured to within manufacturing tolerances of $\pm 0.01 \mathrm{~mm}$.

\subsection{Summary}

This article presents three testcases of specific scenarios to demonstrate the integrated process of 
using CFD and EFD:

(a) the flowfield in a dimple, as could be used on a race-car wing to enhance aerodynamic performance;

(b) the aerodynamics of a wing and wheel, as found on a formula style race-car;

(c) the aerodynamics of high-speed (compressible) flow ground effect situations.

Using both CFD and EFD can result in large datasets, for identical set-ups. Such data are usually spatially located, and take the form of point velocities, temperatures, pressures, turbulent component, etc. scattered throughout some computational domain or experimental region of interest. There are sources of uncertainty and error in both datasets, yet a judgement must be made as to their similarity. Often, the raw field information is compared by 'eye' or pointwise, graphically and in a reasonably qualitative manner. A meaningful and quantitative way of comparing, interpolating and cross-correlating the numerous spatial data would be of great benefit. In the final section of this article, the use of the kriging estimator as a possible mechanism for sensibly combining the two sets of data is outlined. The second case study (the wing and wheel interaction) is used here as an example of the effectiveness of the newly developed method.

\section{CASE STUDIES}

\subsection{Case study 1: wing dimples}

Dimples recessed into a flat plane are of interest due to the ability they have in mixing the boundary layer [15-17]. Previous numerical work has focused on the dimple flow structure along with some limited flow visualization experiments with which to make comparisons. Validation and verification of previous studies lacked consistency in the dimpleo geometry and dimple-based Reynolds number used to reference across numerous investigations by different researchers, along with a verification of the numerical model. In this study, making use of the newly developed tunnel without the moving ground facility, a study was conducted of the effect of adding dimples to ground effect wings in order to enhance the aerodynamic performance. Initially, a study was conducted of the flow in a single dimple, using both LDA and PIV on a dimple with depth $\delta=19.8 \mathrm{~mm}$ and print diameter $D=90 \mathrm{~mm}$, yielding a dimple depth-to-diameter ratio $(\Delta)$ of $\delta / D=\varangle .22$. The Reynolds number based on the dimple print diameter $\left(R e_{D}\right)$ was $9.0 \times 10^{3}$, and the test section height $H / D \approx 3.55$. The experimentally measured velocity in the dimple was compared

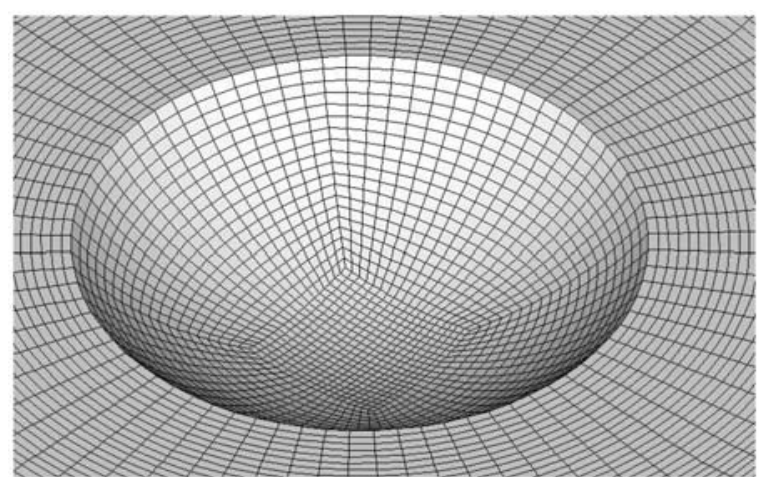

Fig. 5 Fully structured hexahedral mesh on the dimple surface

to a steady-state RANS CFD model, using the identical wind tunnel geometry, Reynolds number, and turbulence intensity. The grid in the dimple comprises fully structured, hexahedral elements throughout the entire domain (Fig. 5). Following the validation of the numerical model, further information about the flowfield was then obtained from the validated results.

A dimple with identical dimensions was used experimentally. The LDA probes were positioned so the beams from the two probes could record three velocity components down to the surface of the dimple without being broken by the spanwise rim of the dimple closest to the probes. Although anodizing the surface matt black assisted with reducing light reflection from the aluminium surface, the curvature of the surface still produced back scattered light which affected the readings up to $2 \mathrm{~mm}$ from the surface.

The nature of the PIV cross-correlation analysis being resolved with a grid of $64 \times 64$ pixels and a 12pixel overlap, results in a 'smoother' time averaged flow field than the LDA, which takes individual point measurements only. LDA captured data over $1.5 \mathrm{~s}$ with an average data rate of $1500 \mathrm{~Hz}$ and peak of $3500 \mathrm{~Hz}$ above the dimple and was limited to 2000 samples at a point; the PIV camera was set to capture 1000 image pairs (over 180 s). Due to the 2D nature of the PIV system, only streamwise and normal velocities are measured. Comparison of the numerically predicted boundary layer and the LDA-measured boundary layer upstream of the dimple shows that the method of matching the inlet boundary condition velocity and turbulence intensity with the experiment, results in greater accuracy (Fig. 6).

The predominant characteristic of the experimental LDA and PIV results (Figs 7 and 8) across the three velocity profile positions for the streamwise flow component is a region of reversed flow beneath the dimple rim from $y / D<\psi-0.05$ which reduces in size and strength as the distance from the leading edge of 


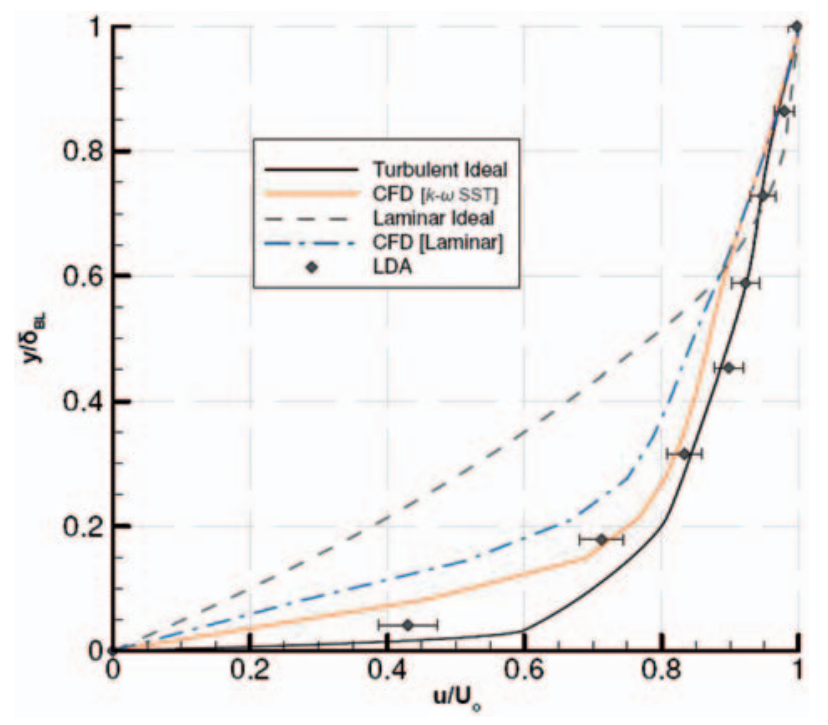

Fig. 6 Boundary layer profile $30 \mathrm{~mm}$ upstream of dimple, $\delta_{B L} \approx 24.3 \mathrm{~mm}$

(a) 02

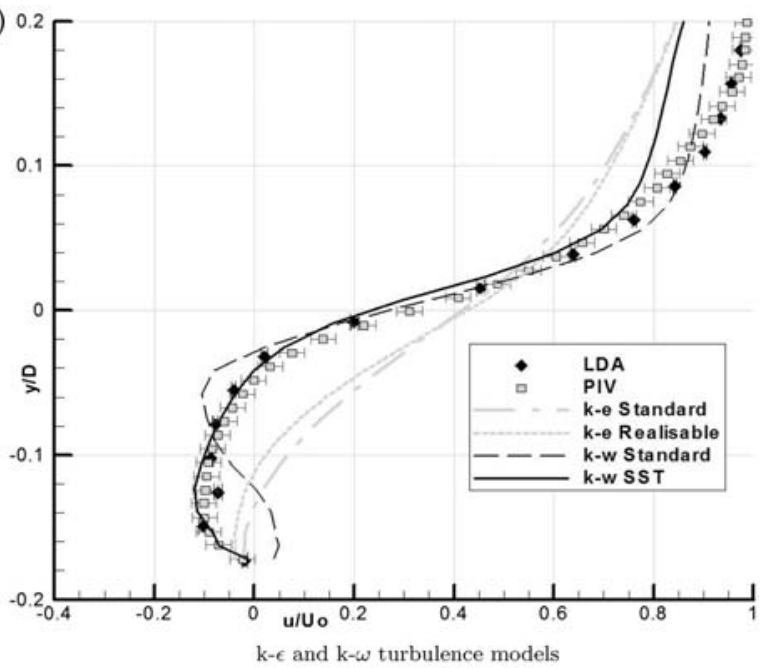

(b) 02

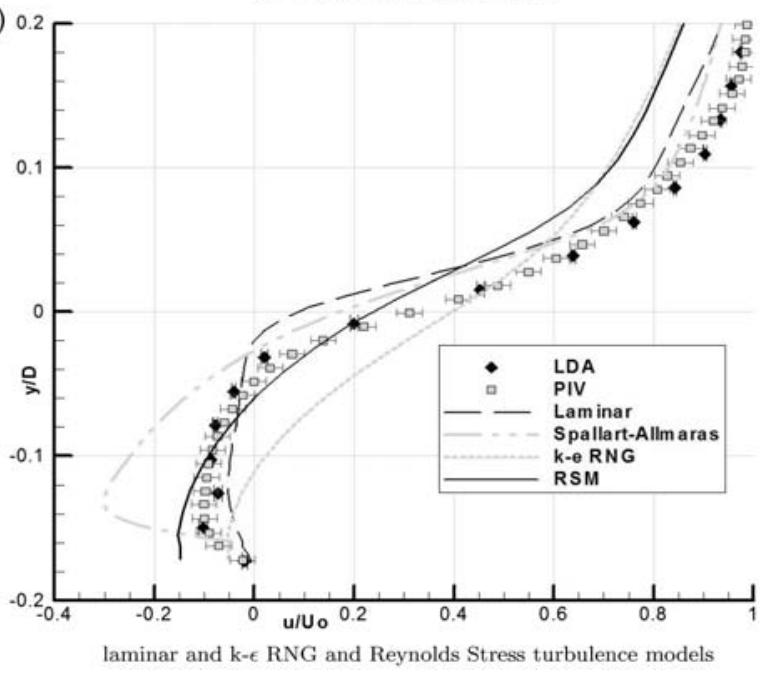

Fig. 7 Position $1 u$ /Uo turbulence model comparison $R e_{D}=9.0 \times 10^{3}$ the dimple increases. The recirculation region lies beneath the initial separated shear layer from the dimple's leading edge. The shear layer at $y / D \approx 0.05$ breaks down as the downstream face of the dimple is approached. The $k-\epsilon$ standard and $k-\omega \psi$ SST turbulence models compare favourably to the LDA and PIV data.

The three versions of the $k-\epsilon$ turbulence model (standard, RNG, and realizable), predict a streamwise velocity profile that is similar to each other. The presiding feature is the minimal reversed flow seen in the LDA and PIV data; in fact, the streamwise flow component is reversed only at position one for $y /$ $D<\psi-0.125$, compared to all three positions for the experimental data. The shear layer is weak, with $u /$ Uo adopting an almost linear profile in positions one, two and three. There is some similarity to the experimental flow profile, but the $k-\epsilon$ turbulence models mostly under-predict the streamwise flow within the dimple.

The laminar model reflects the overall trend of the experimental streamwise velocity profile for all three positions to a better extent than the Spalart-Allmaras model. The reversed flow is slightly less than the LDA and PIV data and indicates more of a large scale 'stalled' flow region beneath the dimple rim. The shear layer that is predicted with the laminar model is also situated higher due to the larger 'stalled' flow region which is in line with the dimple rim $(y / D=0)$ than the actual flow recirculation which sits further beneath the rim of the dimple $(y / D<-0.05)$. The one equation Spalart-Allmaras turbulence model largely reflects the experimental data; however, the initial recirculation in the upstream surface of the dimple indicates a much larger recirculation region, with greater reversed flow than seen in the experimental results. This greater flow recirculation has the resultant effect of locating the shear layer much higher than that measured experimentally.

The streamwise predicted flow profiles for the Reynolds stress model within the dimple initially indicate a reasonable level of agreement beneath the dimple rim $(y / D<\psi)$. However, the shear layer and boundary layer streamwise flow is over-predicted above the dimple rim compared to the LDA and PIV results, and shows poor agreement.

Visualization of the CFD flowfield was then used to attempt to explain the results found from the experimental data (using the $k-\omega \varsigma \S S T$ turbulence model). Figure 9 shows pathlines inside the dimple and it is clear that two lateral vortex cells exist that lie beneath the rim of the dimple. Two symmetric vortex cells are formed at the lower Reynolds number in Fig. 9(a), the core fluid of which is sourced from the upstream half each respective side of the dimple after recirculating 


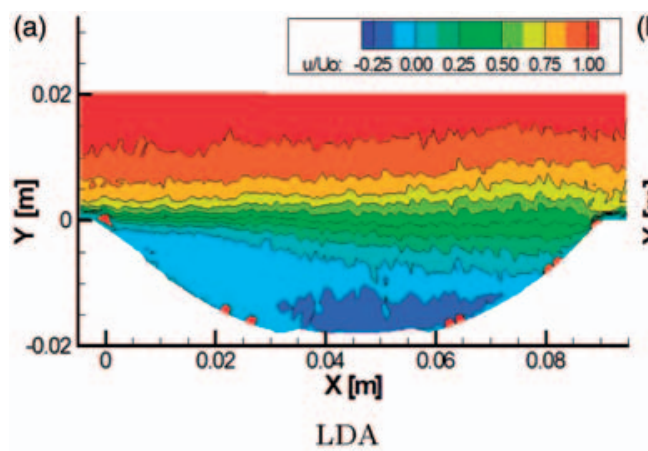

LDA

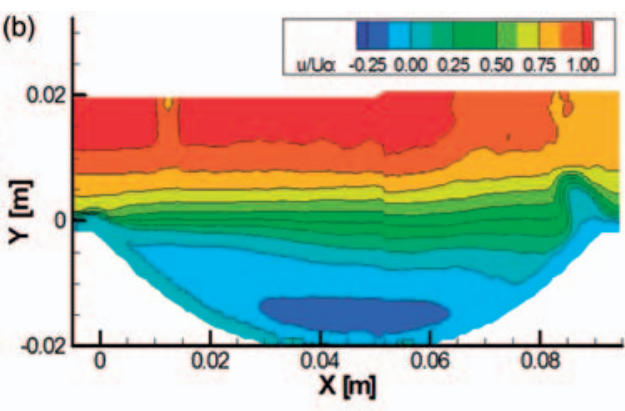

PIV

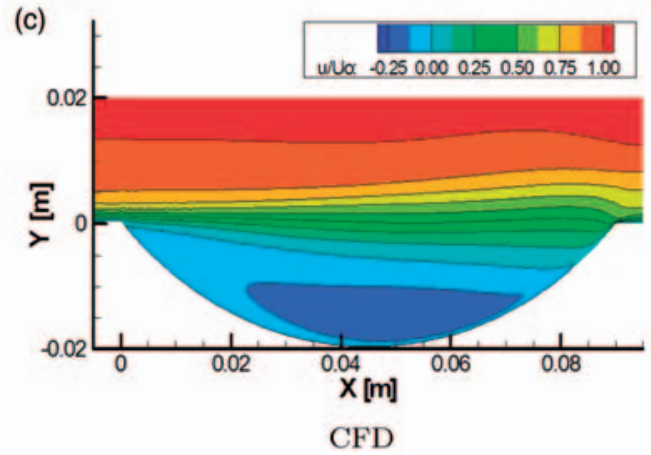

Fig. 8 Normalized streamwise velocity LDA $\operatorname{Re}_{D}=9.0 \times 10^{3}$

(a)

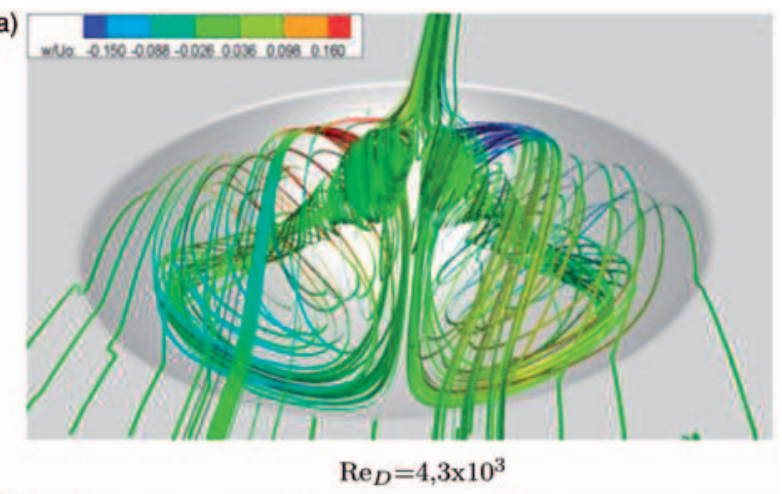

(b)

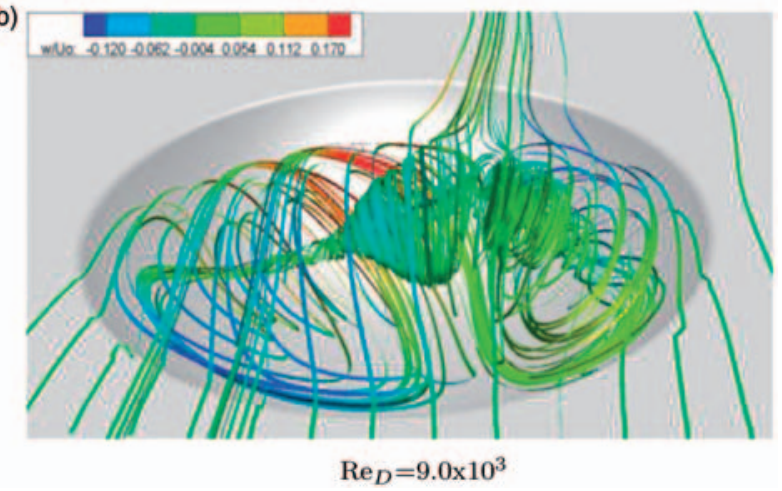

Fig. 9 3D flow field in the dimple

throughout the dimple volume. The effect of increasing the Reynolds number results in an asymmetric flow field in the dimple, with one vortex cell becoming dominant (Fig. 9(b)); noted by Chew and Khoo [18]. Also, evident is the core fluid of the weaker vortex coming from the opposite half of the downstream surface of the dimple which faces the oncoming flow.

The size of the positive and negative spanwise flow regions and the peak positive and negative values are revealed in Fig. 9, as the pathlines are coloured by $w /$ Uo. Due to the stronger vortex cell at the higher Reynolds number increasing the spanwise velocity intensity $(+w)$ on the downstream face of the dimple, fluid is now fed from the downstream face of the dimple into the weaker vortex region. This affects the ability of the vortex in the right-hand half of the dimple to develop with increasing Reynolds number. The increased positive region of spanwise velocity in the dimple limits the volume in which the weaker vortex core can develop, assisting the main vortex to elongate.

This type of flow field information cannot be gained from the experimental analysis; however, the detailed comparison with the available experimental data gives confidence in the numerical results and allows 3D information to be seen.

\subsection{Case study 2: wheel and wing}

Generally, race car aerodynamic studies are focused on individual aerodynamic components such as inverted wings in ground effect [19], diffusers [20], cylinders [21], and exposed wheels [22-24]. In this study, the moving ground wind tunnel and a complementary numerical study was used to study the 
aerodynamic interaction of a Formula One style front wing and wheel.

Two numerical models were developed, one representing the full-scale race-car condition and one representing the wind tunnel. As experiments were not able to be conducted for full-scale conditions, the comparison of wind tunnel results with the corresponding CFD allowed this gap in validation to be filled (Fig. 10). The full-scale computational model was created to reproduce the conditions experienced by a combined wing and wheel operating on a generic open wheel racing car. A full-scale NACA4412 wing and wheel (with chord and diameter of 562.5 and $660 \mathrm{~mm}$, respectively) were modelled with an inlet velocity of $33.3 \mathrm{~m} \mathrm{~s}$, giving a Reynolds number of $1.28 \times 10^{6}$ based on the wing chord and $1.50 \times 10^{6}$ based on the wheel diameter. The NACA4412 profile was selected because of its common use in ground effect aerodynamics studies. The scaled CFD model was developed to replicate the experimental set-up while trying to maintain as many of the features of the full-scale computational model as possible; the mesh used is shown in Fig. 11. The wing chord and the wheel diameter are equal to that of the wind tunnel models (75 and $88 \mathrm{~mm}$, respectively, giving a scale of 1:7.5). The wind tunnel experiments (and corresponding CFD simulations) were conducted at a Reynolds number of $5.11 \times 10^{6}$ using the wing chord as the reference length (or $5.98 \times 10^{6}$ based on the wheel diameter). The boundary layer was not tripped to promote transition in either the computational or experimental simulations.

(a)
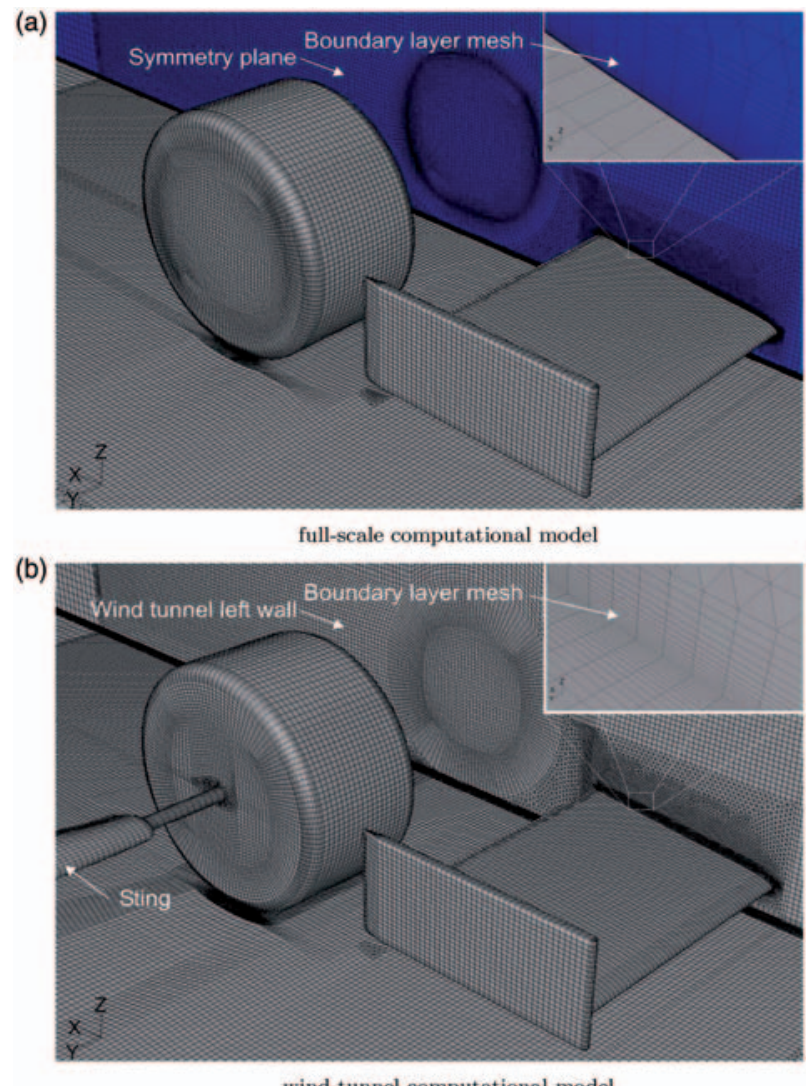

Fig. 11 Surface mesh indicating grid structure

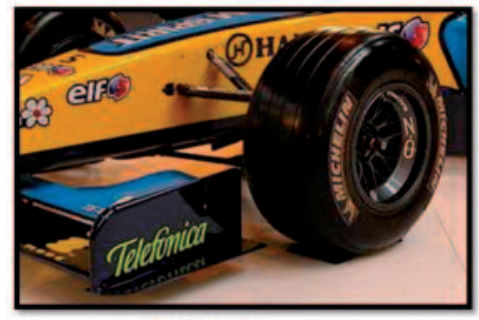

REALITY

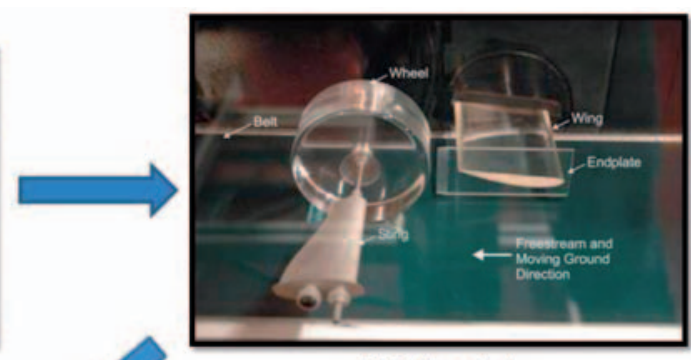

EFD (low Re)

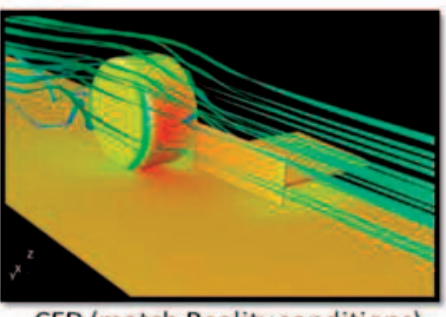

CFD (match Reality conditions)

CFD (match EFD conditions)

Fig. 10 Description of methodology 
Results obtained with several different turbulence models were compared to LDA measurements made in the wake of the wing and the wheel in order to determine the most suitable turbulence model and to demonstrate that the computational model can accurately reproduce the variations in flow structures associated with changes in the wing span and angle of attack of the wing (parameters that were investigated in a further wing and wheel interaction study). An example is given in Fig. 12, showing the $x$-velocity on the $x / c=4.75$ plane (just downstream from the wheel - see Fig. 19 later in this article for a further representation), where $c$ is the wing chord.
LDA results (Fig. 12(a)) indicate that the wheel wake is directly positioned behind the wheel and consists of two upper, counter-rotating vortices formed by the separation from the top of the wheel tread. Above the wheel, the flow is being entrained into the wheel wake and in the process a downward velocity component in the centre of the wake is created. The wing wake can be observed between the wind tunnel wall and extending across to the inner face of the wheel. Within the wing wake, two additional counter rotating vortices can be observed. While all efforts were made to obtain very detailed experimental data, certain regions could not be measured due to (a) Experimental LDA results

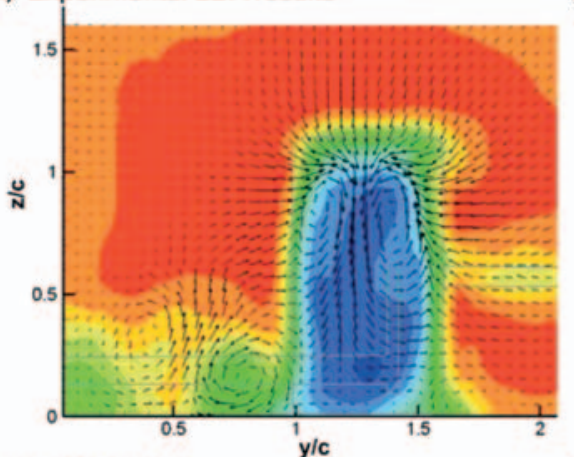

(c) $k-\varepsilon$ Standard

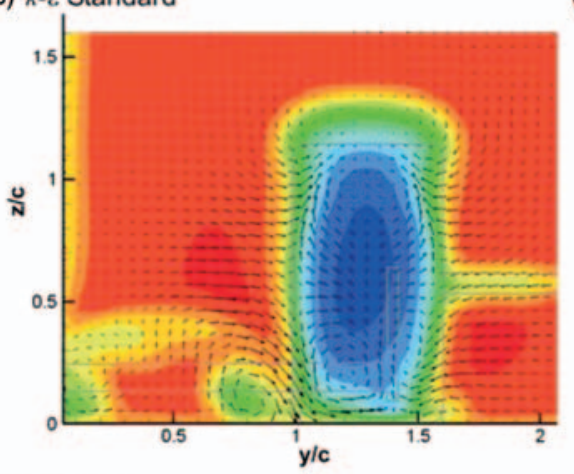

(e) $k-\varepsilon$ RNG

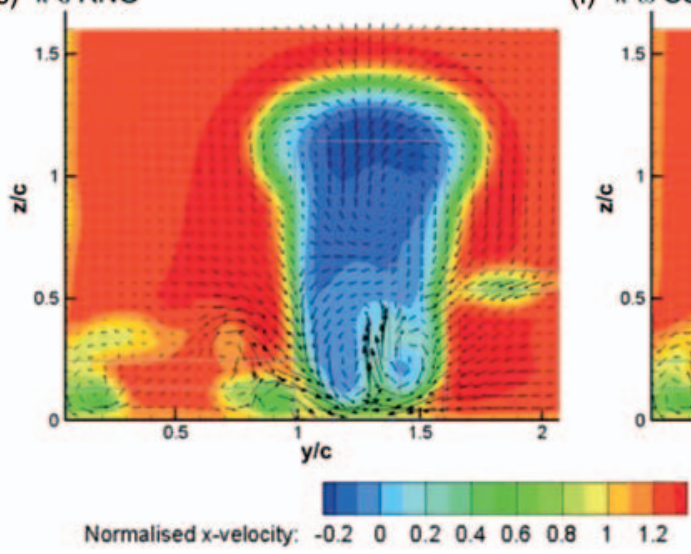

(b) Spalart-Allmaras

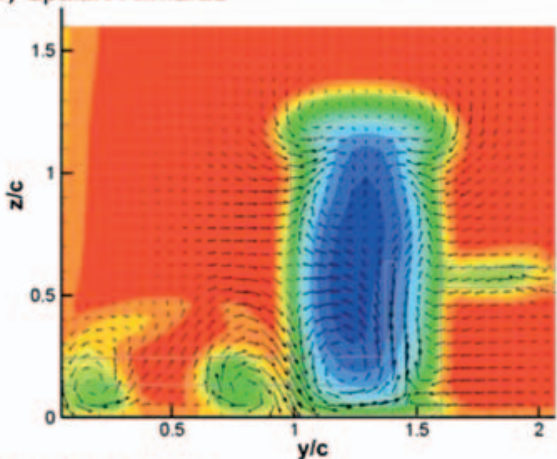

(d) $k-\varepsilon$ Realizable

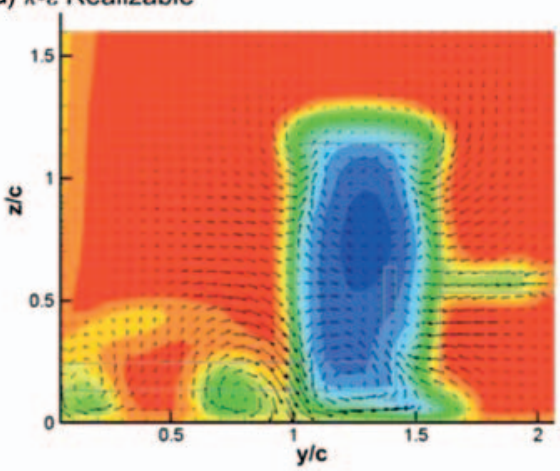

(f) $k-\omega \mathrm{SST}$

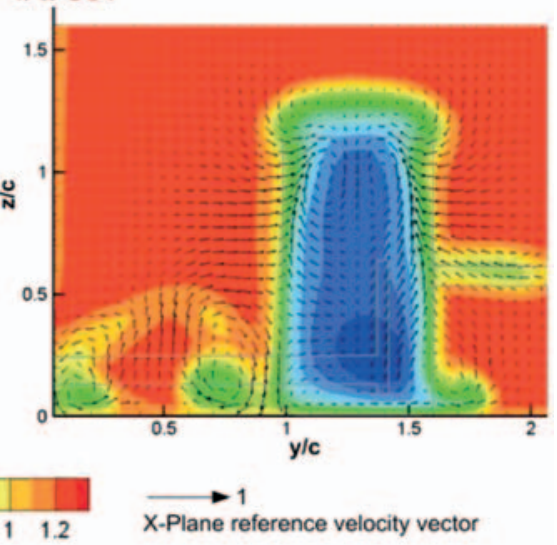

Fig. 12 Normalized $x$-velocity and in-plane comparisons to LDA results on $x / c=0.75$ plane for the wind-tunnel situation 
geometric constraints. By comparing with a range of numerical simulations (at the points where detailed measurements could be obtained) different CFD models could be evaluated, and the most appropriate for this particular case determined. The chosen numerical model could then be used to find the full flowfield.

A comparison was made with a number of numerical solutions, in which the difference between each is the method of turbulence closure used. Comparing to results obtained with the Spalart-Allmaras model indicates that this turbulence model over predicts the height of the wheel wake by a value of $0.1 c$ and that the two upper vortex structures are positioned slightly lower and closer to the centre of the wheel wake. The largest and the least realistic wheel wake structure was calculated with the $k-\epsilon$ RNG turbulence model, which suggests that the wheel wake is narrower at the base than the top. No upper wheel wake vortices were obtained with this turbulence model and instead two vortices were formed adjacent to the ground causing an upwash in the centre wheel wake.
It appeared that either the $k-\epsilon$ SST and $k-\epsilon$ realizable turbulence models could be considered suitable for use during a computational study of the wing and wheel interaction. Numerous computational results were obtained for a combination of varied wing span, angle of attack, and height as well as wheel widths and tracks, for the wind tunnel case and the real life situation [25]. Doing so indicated that three main wing and wheel interactions may occur depending on the combination of wing and wheel parameters chosen. The main variation between the three states is dependent on the path that the main and secondary wing vortices take around the wheel and subsequently how they interact with the wheel wake.

The three alternatives paths that the wing vortices may take were determined to be as follows (Fig. 13).

1. Both main and secondary wing vortices travel outboard of the wheel (interaction a).

2. The secondary wing vortex travels outboard of the wheel while the main wing vortex is drawn inboard (interaction b).

(a) Both wing vortices travelling outboard of wheel
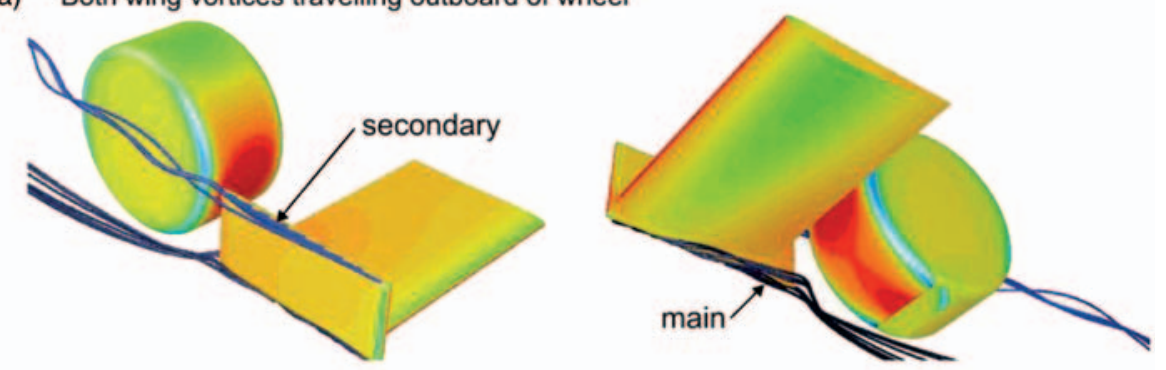

(b) Main wing vortex travelling intboard while secondary vortex travels outboard of wheel
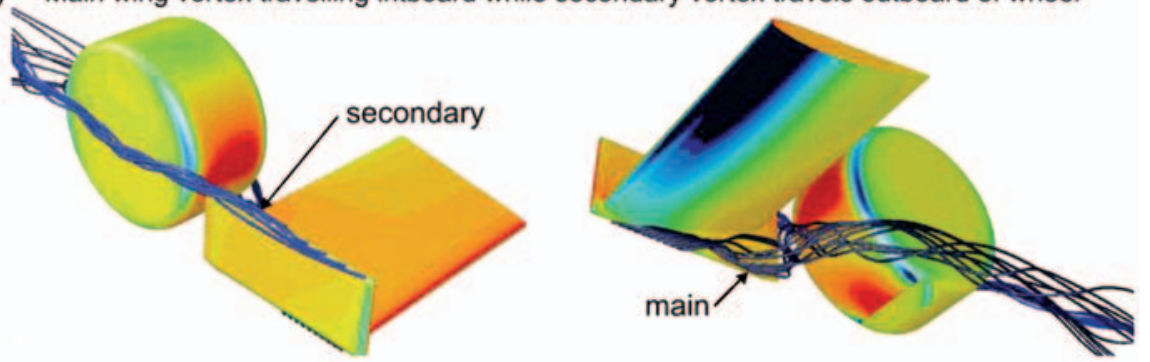

(c) Both wing vortices travelling inboard of wheel
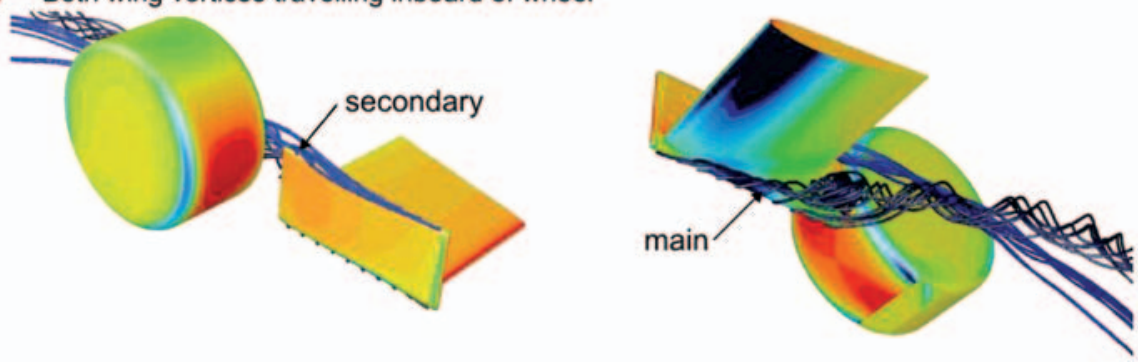

Fig. 13 The three wing and wheel vortex interactions 
3. Both the main and secondary vortices travel inboard of the wheel (interaction c).

Interaction 'a' was observed to occur only when the span was equal to the wheel width and the wing angle of attack was less than or equal to 4 . Due to the wing spanning across the entire wheel, the high-pressure regions formed forward of the wheel reduced the wing's ability to generate suction beneath the wing and reductions in wing downforce and drag of up to 40 percent and 70 percent, respectively were obtained in this configuration. Increasing the angle of attack to a value of 8 or greater at a span equal to the wheel track allowed interaction ' $b$ ' to be obtained. Doing so increased the low-pressure region beneath the wing sufficiently to allow the high-pressure regions formed forward of the wheel to be overcome, such that the main wing vortex travelled inboard of the wheel. As a result, the wing's ability to generate downforce was generally found to be less affected with downforce reductions of 10 per cent been obtained in comparison to an equivalent wing in isolation. The main wing vortex interaction with the wheel wake also reduced the downwash experienced in the central wheel wake region allowing the wheel lift and drag to be reduced by up to 45 percent and 25 percent, respectively. Reducing the angle of attack of the $S / c=4.24$ wing to 0 and 4 allowed the secondary vortex to travel inboard of the wheel allowing interaction 'c' to occur. The highpressure region forward of the wheel was found to increase the pressure differential experienced about the bottom edge of the endplate allowing the main wing vortex to be stronger and therefore assist with alleviating the separation that would be experienced by the equivalent wing in isolation. When this was observed, wing downforce and drag increases of up 10 per cent and 20 per cent, respectively, were observed.

\subsection{Case study 3: compressible ground effect aerodynamics}

Two different scenarios were investigated - that of a NATO 5.56-mm projectile fired in close proximity to a ground plane at Mach 2.4, and that of an RAE2822 wing section at super-critical subsonic Mach numbers (in particular a case at approximately Mach 0.65). The two methods for high-speed ground simulation examined in more detail here are the elevated ground plane, which reduces the extent of the boundary layer, and a symmetry ground condition, which in the experimental case requires two identical models. Wind tunnel experiments with these model arrangements, using pressure tappings and schlieren photography have been carried out [26] and supplemented by numerical simulations of the experiments in order to better quantify the relative merits of each method.

In the case of the projectile, the elevated ground was designed to extend far enough upstream of the projectile (approximately $140 \mathrm{~mm}$ ) such that any disturbances generated by the leading edge of the ground would not impinge upon the projectile. The boundary layer grows naturally from the leading edge of the elevated ground, giving it a thickness of $2.5 \mathrm{~mm}$ at the leading edge and $3.8 \mathrm{~mm}$ at a point coincident with the projectile base. This accounts for a large proportion of the gap between the projectile and ground. At low height/diameter $(h / d)$ clearances, the boundary layer on the elevated ground is significant in relation to the size of the test model, as evidenced in Fig. 14, in particular downstream of the impingement of the bow shock generated by the projectile model. Figure 14 indicates that at the projectile base the ground boundary layer has increased to approximately $3.8 \mathrm{~mm}$, which corresponds to about 45 percent of the distance $h$ in this flowfield. The symmetry method produced results (in terms of pressure distribution for all cases examined) closer to that of an ideal moving ground than the elevated ground plane. It would therefore be recommended for all conceivable wind tunnel studies of supersonic ground effect. The presence of any ground boundary layer noticeably distorts the flow downstream of the first shock/ ground interaction. It could still be beneficial however, to use the elevated ground method, in particular if the ground plane were extensively instrumented or thermochromic liquid crystals were used [27]. This would provide some potentially useful information about the 'footprint' of the shocks propagating away from the model which could not be obtained with the symmetry method. There may also be cases in which the modifications of the pressure distribution caused by the boundary layer on the ground plate have a negligible net effect on the resulting aerodynamic forces, but this would have to be verified for each application.

The situation is now extended to transonic ground effect, to determine if the symmetry method is also a feasible approximation for this more complex aerodynamic regime. The transonic wind tunnel of the US Naval Academy in Annapolis, Maryland was used for the transonic tests. The tunnel features a test section of cross-sectional area of $0.0418 \mathrm{~m}^{2}$, with dimensions of $0.2057 \times 0.2032 \mathrm{~m}^{2}$. Tests were made with the porous walls partially open, and fully closed, the latter of which were used for most of the experiments in order to facilitate more reliable CFD modelling of the test conditions with simple boundaries, as is commonly recommended (but seldom implemented) for any code validation of this nature [28]. The wing is an RAE2822 section - commonly used in transonic 
(a)

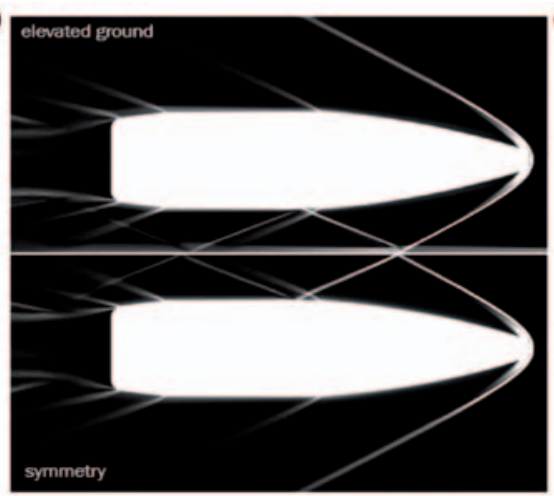

CFD: symmetry and elevated ground methods

(c)

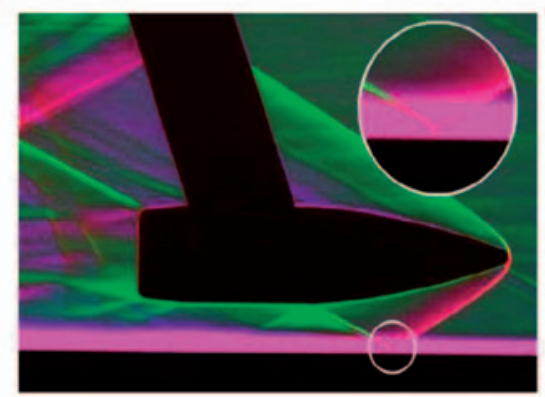

Experimental colour schlieren of elevated ground test, $\mathrm{h} / \mathrm{d}=0.5$ (inset, symmetry method test, $\mathrm{h} / \mathrm{d}=0.52$ (inset, shock/boundary layer interaction detail) crossing shock interaction detail)

Fig. 14 Comparisons of different modelling scenarios

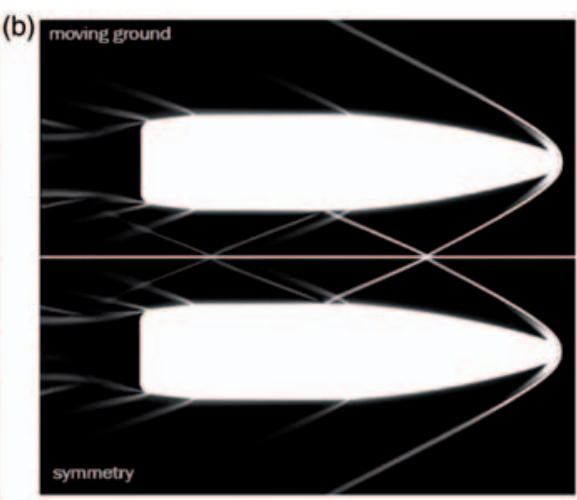

CFD: symmetry method and a moving ground

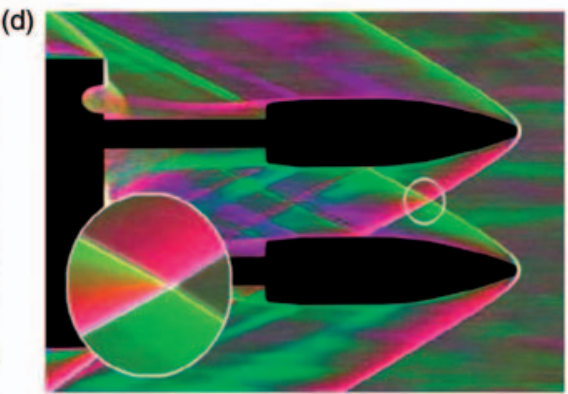

Experimental colour schlieren of ) 
flow, and is much more pronounced for the elevated ground method. The peak upper surface Mach number is markedly increased, and the flow is nearsonic in the elevated ground case. This indicates that at a freestream Mach number slightly higher than that of this particular experiment, an upper surface shock would have formed with the elevated ground, where it would not have with the symmetry method. The flow is also increasingly accelerated in the gap between the wing and the ground, such that the lower surface shock in the elevated ground case is stronger than that observed with the symmetry method, as illustrated in the pressure distributions at the mid-span in Fig. 16. This causes a fairly strong shock/boundary

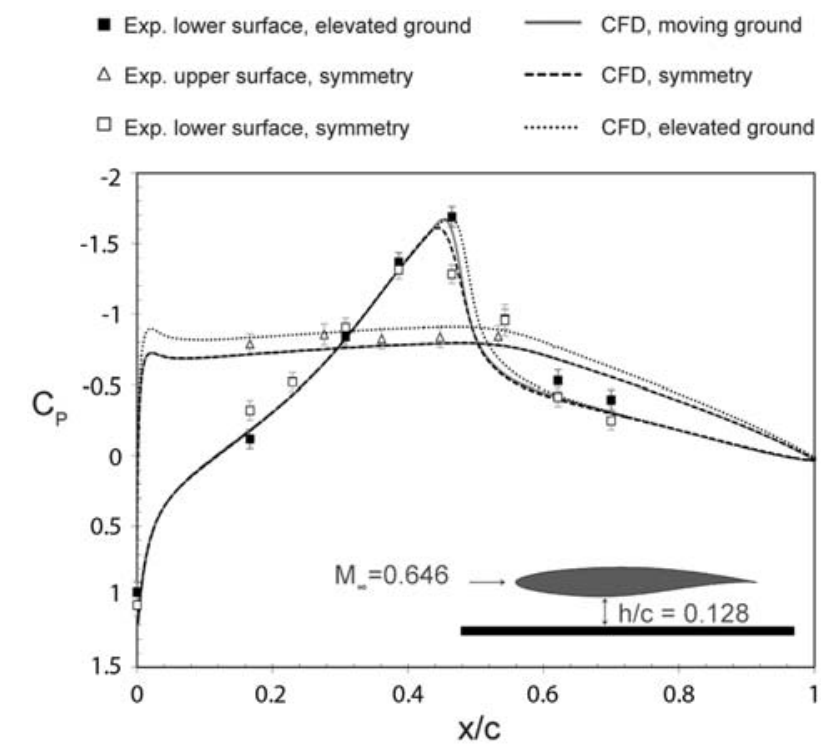

Fig. 16 Time-averaged pressure distribution $C_{p}$, comparisons of experimental symmetry, moving ground, and elevated ground methods to CFD, at the mid-span plane on the wing layer interaction on the elevated ground, which immediately thickens the boundary layer there.

Ground plane pressure distributions are presented in Fig. 17, allowing the character of this interaction to be examined. The symmetry and elevated ground methods show a strong shock presence and accompanying pressure rise at close to $x / c=\theta .5$, with the elevated ground exhibiting a slightly diffused version of this interaction due to the 'cushioning' effect of the boundary layer. The downstream effect of this is such that the elevated ground pressure distribution does not recover to the same extent as the other two, near-identical pressure distributions, and would therefore exert a lingering influence into the wake region. The symmetry method does not quite capture the extent of the suction peak, and thus a clear connection between very small ground clearances and increasing discrepancies between the symmetry and moving ground boundary representations emerges. Interestingly, although the elevated ground plate clearly causes a large, unwanted disruption to the flow at the leading edge, the predicted ground pressure distribution in the CFD soon aligns with the symmetry and moving ground distributions. The dominant pressure gradient produced by the actual wing therefore seems to have a useful 'settling' effect on the leading edge flow, though the influence of the elevated ground on the upstream flow remains.

The scaling of tests from wind tunnel to actual flight a challenge in aeronautics regardless of ground proximity, and at small scale is enhanced for ground effect problems due to the proportionally large-body boundary layer size with respect to the ground clearance. If the elevated ground is used, its boundary layer contributes further to this problem, as already seen. Assuming a higher Reynolds number is achieved by directly scaling up the model for testing

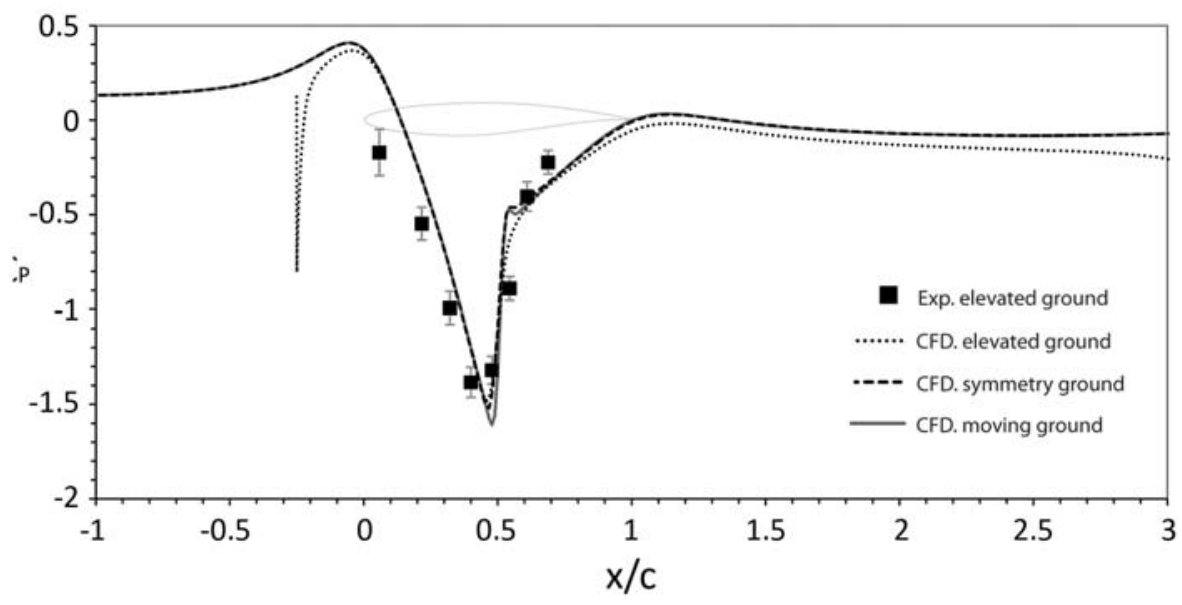

Fig. 17 Time-averaged pressure distribution $C_{p}$, comparisons of experimental symmetry, moving ground and elevated ground methods to CFD at the mid-span plane on the ground plane 
in a larger facility, the symmetry method can suffer from a large blockage ratio compared to an elevated ground which can remain fairly slim as it is scaled up. The choice of method for testing may, then, depend on the size of the test section, at least up until nearsonic freestream Mach numbers, where the symmetry method is the only one which could be relied upon due to the distortion of the flow caused by the leading edge of the elevated ground plane. As a brief examination of the applicability of small-scale testing with the symmetry method for flows of a Reynolds number ten times higher than those of the experiments, CFD was re-run with appropriately scaled versions of the case previously seen in Fig. 16.

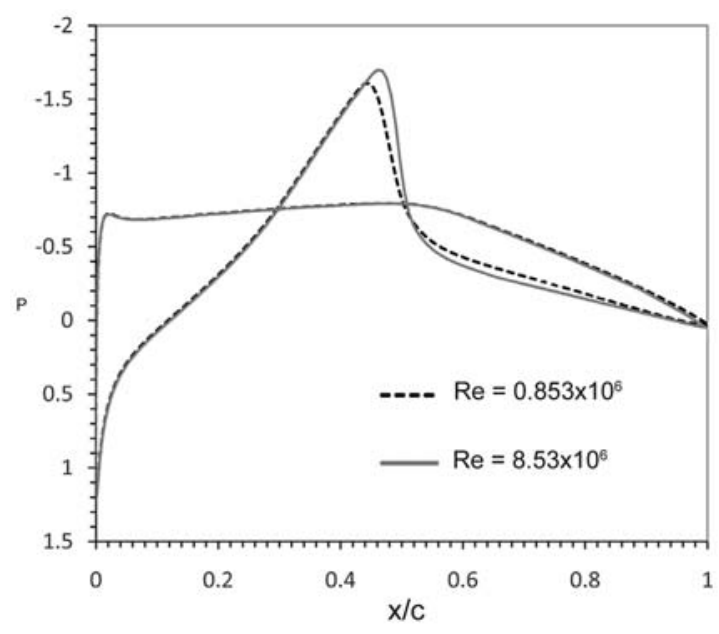

Fig. 18 Time-averaged pressure distribution for the symmetry ground method
The pressure distributions obtained, as shown in Fig. 18, indicate that the primary consequence of the increase in scale was to push the shock wave further back along the chord by around $x / c=0.03$. While the general distribution is still similar, it can be reasoned that, for certain crucial points in the evolution of the flowfield with respect to increasing Mach number or decreasing ground clearance, the smallscale tests could provide misleading results. This would be particularly likely in the case of determination of the exact critical Mach number, or the onset of buffeting flow. It is important to note that the discrepancies shown in the two results shown are of similar order to the differences observed in predictions obtained by use of different turbulence models in initial comparisons. Those comparisons in themselves could not have been made without useful experimental data, and thus it is clear that only when experimental and numerical analysis are conducted in close partnership, the greatest levels of insight and confidence can be obtained from the research.

\section{CORRELATING EFD AND CFD DATASETS}

If two sets of spatial data correlate well, it should be possible to improve the prediction of one variable by considering its statistical correlation with the other variable. In any good fluid dynamics study involving the integrated use of CFD and EFD, such datasets will be readily available.

The kriging estimator was originally developed in the earth sciences by Krige [30] and later formalized

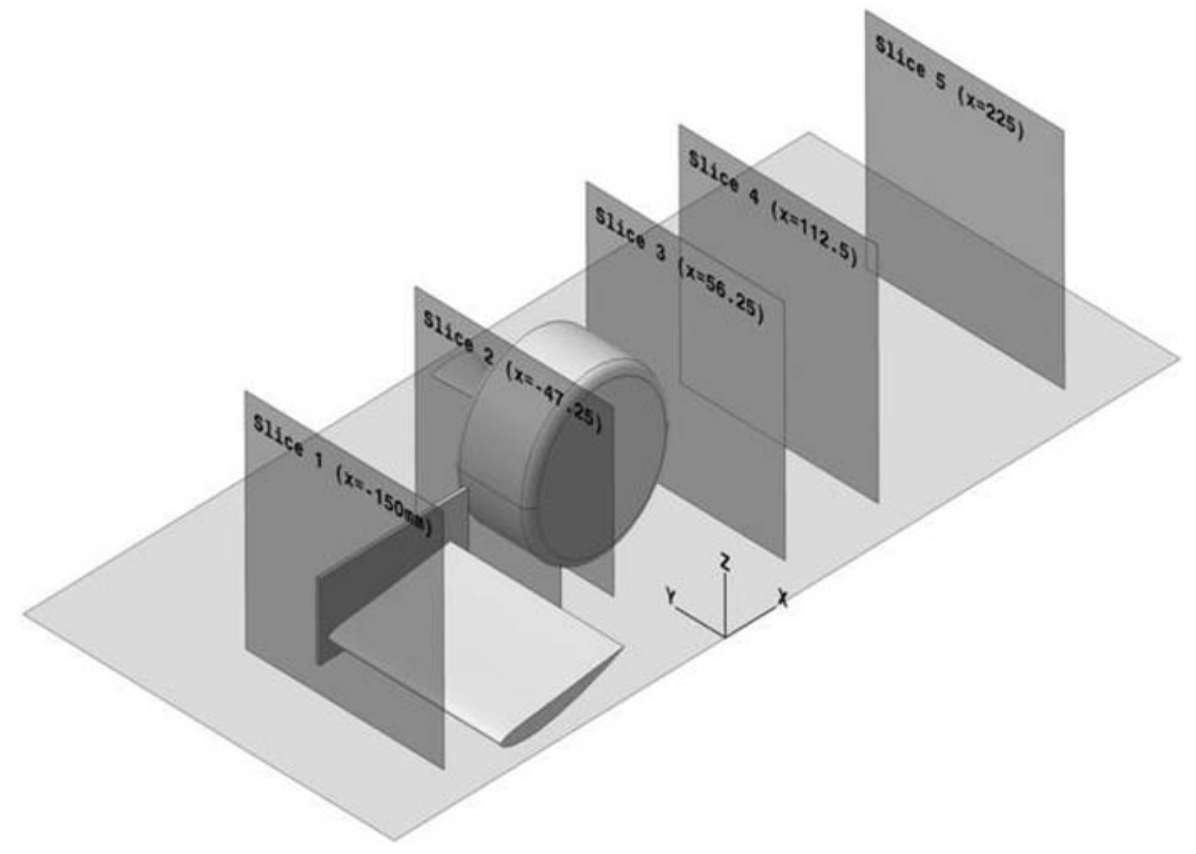

Fig. 19 Measurement planes 
by Matheron [31], to estimate the extent and quality of underground ore reserves from limited point (borehole) surveys, a discipline known as geostatistics. In the area of numerical analysis, the kriging estimator has received attention in the past for the construction of notionally optimal response surfaces to leverage numerically expensive information [32]. It is essentially a technique for spatial interpolation that fits a stationary random function to a set of sampled known point values, thus producing a response surface. The interpolates are weighted averages of the point values, and the weights are calculated in such a way as to minimize the modelled kriging variance of the estimate [33]. The kriging variance itself is representative of the modelled uncertainty of the estimate at some remote point, and it is dependent upon the statistical continuity of the data. Kriging has a number of advantages as an estimator. It is the best linear unbiased estimator (BLUE) and without a socalled nugget effect, it will construct a response surface that exactly interpolates the known data. Cokriging extends the basic interpolation capabilities of kriging to multiple correlated datasets.

Kriging can be viewed as a non-parametric interpolator - it fits a response surface through a given set of known data points by assigning weights to the data. However, unlike other common non-parametric interpolation methods, its basis functions derive from an underlying statistical model. An attempt is made to characterize the smoothness (spatial continuity) of the complete phenomena based on such nodal data as are available for interpolation and to this end, covariance functions of the data are estimated, so that a model for spatial continuity may be determined. There are two steps for estimation. The first is structure identification, which informs the theoretical random function model, and the second is estimation, in which this model is used to form a covariance matrix which may be solved for the linear weights. A particular random function is notated $P^{\alpha}$. A random function $P^{\alpha \psi}$ is characterized by its covariance function; $C_{\alpha \alpha}(x, y)$. This expresses the covariance between the regionalized random variables $P^{\alpha}(x)$ and $P^{\alpha}(y)$, which measures the strength of the relationship between data located at $x$ and data located at $y$. In the context of estimation, this provides a means of gauging the relative importance of the surrounding data, and is appropriate where data is spatially dependent. Fundamentally, it is assumed that the spatial data for interpolation are a realization of a given random function - a regionalized random variable. If there are secondary data that are expected to be strongly correlated to the primary variable, it should be possible to improve the estimate by its consideration. In this case, the concepts are extended by introducing cross-covariance functions $C_{\alpha \beta}(x, y)$. The cross-covariance $C_{\alpha \beta}(x, y)$ denotes the covariance between $P^{\alpha}(x)$ and $P^{\alpha}(y)$, where 'Cov' indicates the usual covariance function

$$
C_{\alpha \beta}(x, y)=\operatorname{Cov}\left(P^{\alpha}(x), P^{\beta}(y)\right)
$$

Here, the developed kriging algorithm has been applied to the wing and wheel study discussed earlier (section 2.2). The velocity components are considered as independent, Gaussian, realizations of a random function with an unknown drift. The
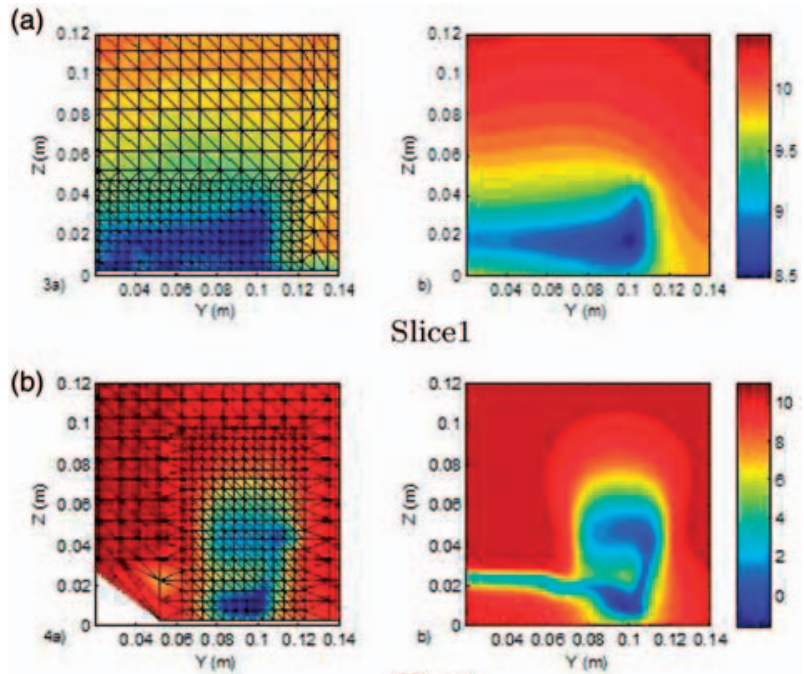

Slice1
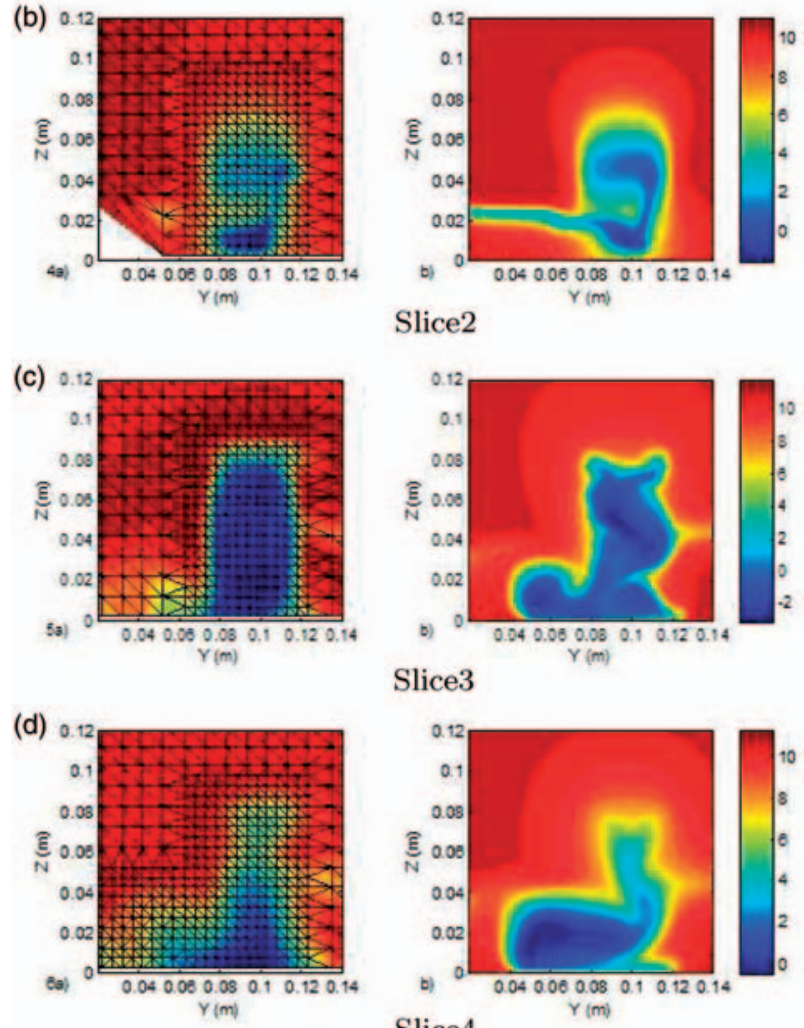

Slice3
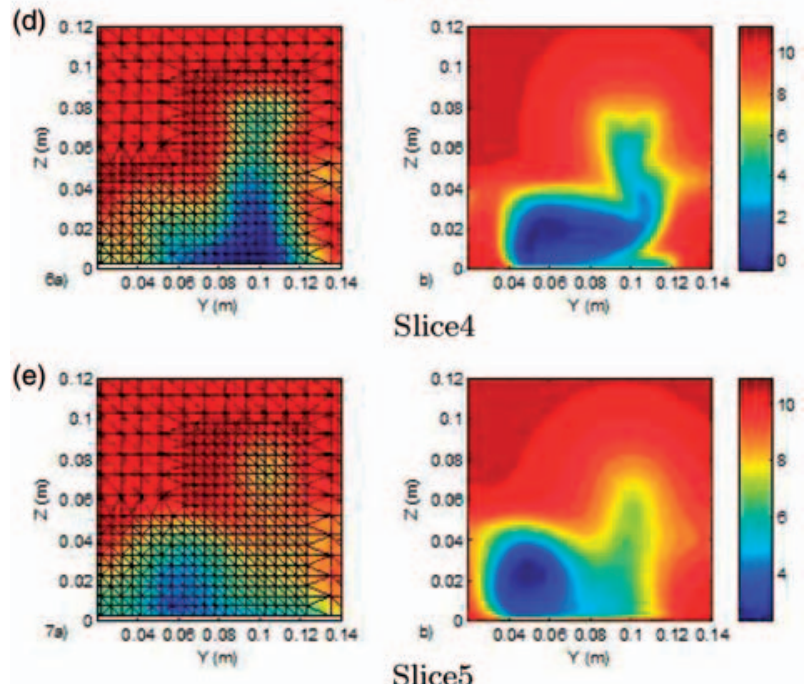

Slice 4

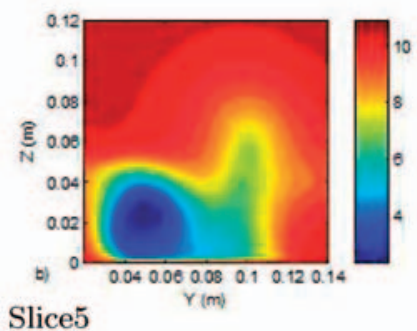

Fig. 20 Raw $x$-velocity for LDA and CFD 

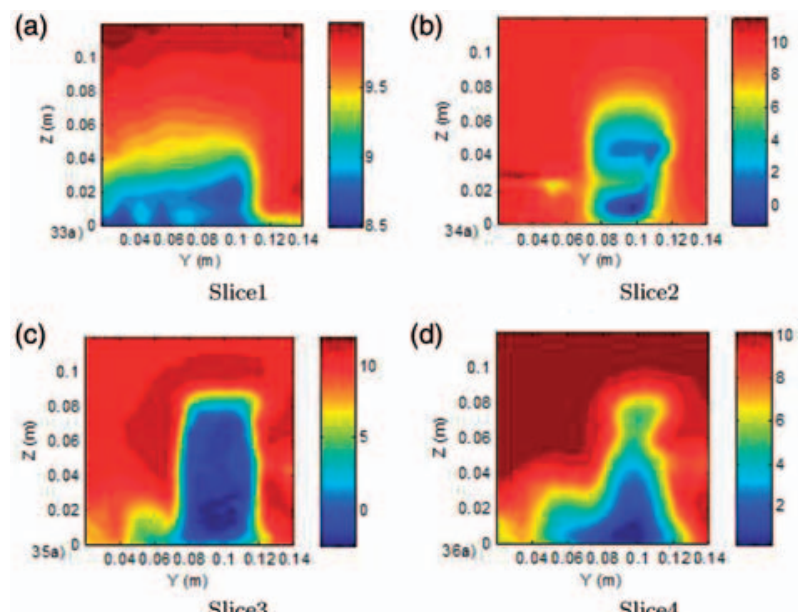

(d)

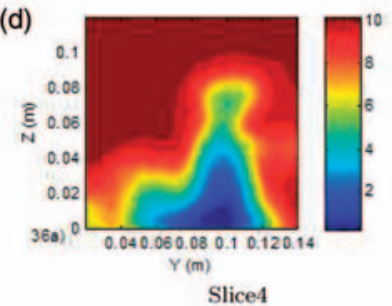

(e)

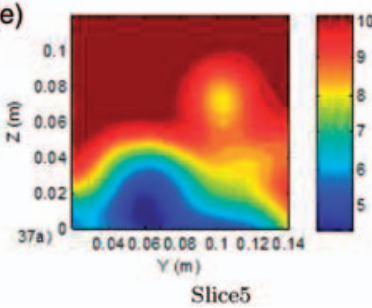

Fig. 21 LDA estimates cokriged with CFD

techniques of Universal Kriging are used to estimate the drift and structure identification proceeds along the lines described under 'Model for covariance'. The primary function of kriging is to produce an interpolated response surface from nodal values.

The original data are shown in Fig. 20, where contours of $x$-velocity are shown, with experimental (LDA) results on the left and CFD on the right. The LDA images also show the measurement grid used. The five images represent the results at five different measurement planes, as shown in Fig. 19.

The cokriged results (Fig. 21) clearly show that the wake behind the wheel can be blended into the experimental result because of the high spatial correlation of the numerical and experimental results. Furthermore, there is a modified interaction with the moving ground underneath the wing which is edited in, as well as a more pronounced boundary layer at the moving ground under the stagnation region in front of the wheel.

The cokriging at slice three is also modified by the extra data, if somewhat less dramatically. The small wake behind the sting outboard (to the right) of the wheel wake has been sampled too coarsely by the LDA to resolve it properly; however, the structure is resolved with more clarity when the numerical result is incorporated.

It can be seen in Fig. 20 that the region inboard of the wheel wake is also sampled coarsely, which means that in the cokriged estimates Fig. 21, a number of structures are modified. The vortex inboard of the wing is amplified as it is well described by the extra numerical data. There is a strong boundary layer at the bottom of Fig. 21 as the moving ground is approached. Experimental data are relatively sparse (and in any case unreliable) close to the walls and boundaries of the wind tunnel, but numerical data must resolve the boundary layer whether by wall functions or local mesh refinement. The cokriging blends these two extremes.

There is also some modification to the region of low $x$-velocity directly in the wake of the wheel shown in the third slice, wherein the rather intricate vortex structure predicted by the numerical work is now faintly evident.

There remains much work in extending the capabilities of the current algorithms for larger and more demanding problems, thus creating a closed-form post-processing tool. However, it has been shown that the proposed techniques can be used to provide a better picture of the overall results correlation, and can also be used to provide 'best' overall estimates of important variables.

\section{CONCLUSIONS}

Examples of ground effect aerodynamics research have been described as a way of demonstrating the successful and synergistic integration of CFD and EFD. Experimentally, a moving ground wind tunnel is used for much of the work, and the development of this facility is itself an example of the integrated use of CFD and EFD to produce the desired outcome. Nonintrusive measurement techniques are used, with the advantage that these techniques also produce large amounts of field data (for better comparison with CFD results).

A study was conducted of the flow within a single dimple. This was a preliminary study that then allowed further investigation into the effect of adding dimples to ground effect wings in order to enhance the aerodynamic performance. The experimentally measured velocity in the dimple was compared to a steady-state CFD model, using the identical wind tunnel geometry, Reynolds number and turbulence intensity. Following the validation of the numerical model, further information about the flow-field was then obtained from the validated results, which were unable to be seen from the experimental data alone. Experimental results suggested certain flow behaviour which was subsequently studied numerically.

Second, the aerodynamic interaction of a Formula One style front wing and wheel was studied, with two CFD models developed, one representing the fullscale race-car condition and one representing the 
wind tunnel. As experiments were not able to be conducted for full-scale conditions, the comparison of wind tunnel results with the corresponding CFD allowed this gap in validation to be filled. Results obtained with several different turbulence models were compared to LDA measurements made in the wake of the wing and the wheel in order to determine the most suitable turbulence model and to demonstrate that the computational model can accurately reproduce the variations in flow structures associated with changes in the wing span and angle of attack of the wing (parameters that were investigated in a further wing and wheel interaction study).

A different facility was used for the third example, as in this case supersonic and transonic ground effect aerodynamics was studied. The CFD modelling was an integral part of this process, as the creation of a moving ground in CFD is a trivial task but definitely not so experimentally. Two alternative methods of simulating the ground were considered: the elevated ground plane, which reduces the extent of the boundary layer, and a symmetry ground condition, which in the experimental case requires two identical models. Wind tunnel experiments were carried out for both the supersonic and transonic cases, and compared to numerical models of the wind tunnel situations as well as the ideal-case moving ground scenario. During the experimental program, it became clear that 3D effects were substantial and subsequent numerical models were run as full 3D cases.

Finally, in order to intelligently analyse all the data obtained through the different computational and experimental processes, it has become increasingly useful to have a tool that can achieve this. In this study, the kriging estimator was shown to provide a method for integrating two datasets and, beyond just comparing them, using one to improve the other.

In each case described here, the computational and experimental studies were performed by the same researcher, with each component of the study informing the other, representing a truly synergistic CFD and EFD study. It is also critical to compare to reliable published data from other researchers during each project; researcher bias and validation errors (e.g. an error in using incorrect geometry could be followed through in both CFD and EFD studies) can then be minimized.

\section{FUNDING}

This research received no specific grant from any funding agency in the public, commercial, or notfor-profit sectors.

\section{REFERENCES}

1 Cummings, R. M. and Knowles, K. Guest editorial. Proc. IMechE, Part G: J. Aerospace Engineering., 2009, 223, i-ii.

2 Doig, G., Barber, T., Neely, A., and Myre, D. Experimental validation as an integral component of computational fluid dynamic research. ANZIAM J., 2010, 51, C265-C279.

3 Barber, T., Doig, G., Beves, C., Watson, I., and Diasinos, S. Keynote lecture: running on all cylinders - the integration of CFD and EFD for ground effect aerodynamics studies. In Symposium on Integrating CFD and experiments in aerodynamics, The von Karman Institute for Fluid Dynamics, Brussels, Belgium, 14-16 September 2009.

4 Barber, T. J. Aerodynamic ground effect: a casestudy of the integration of CFD and experiments. Int. J. Vehicle Des., 2006, 40(4), 299-316.

5 Barber, T. Technical note on the appropriate CFD boundary conditions for the prediction of ground effect aerodynamics. Aeronaut. J., 1999, 103(1029), 545-547.

6 Barber, T., Leonardi, E., and Archer, R. Causes for discrepancies in ground effect analyses. Aeronaut. J., 2002, 106(1066), 653-657.

7 Fago, B., Lindner, H., and Mahrenholtz, O. The effect of ground simulation on the flow around vehicles in wind tunnel testing. J. Wind Eng. Ind. Aerodyn., 1991, 38, 47-57.

8 Lamb, J. Critical velocities for rocket sled excitation for rail resonance. Johns Hopkins APL Tech. Digest., 2001, 21 (3), 448-458.

9 Purdon, J., Mudford, N., and Kleine, H. Supersonic projectiles in the vicinity of solid obstacles. In Proceedings of the 27th International Congress on High-Speed Photography and Photonics, SPIE, Bellingham, Vol. 6279, 2007, p. 627929.

10 Diasinos, S., Barber, T. J., Leonardi, E., and Hall, S. D. The Validation of a 2D CFD model for the implementation of a Moving Ground in a UNSW Wind Tunnel. In Proceedings of the 5th Pacific Symposium on Flow visualization and image processing (PSFVIP5), Daydream Island, Australia, 27-29 September 2005, Published on CD-Rom, Paper 3.2, 12 pp.

11 Barlow, J. B., Rae, W. H., and Pope, A. Low-speed wind tunnel testing, third edition, 1998 (John Wiley and Sons, New York).

12 Motson, B. C. and Archer, R. D. Design features of the low speed $4 f t x 3 f t$ return circuit wind tunnel, report no. 19969/FMT/3, 1969 (University of New South Wales, Australia).

13 Roache, P. Verification and validation in computational science and engineering, 1998 (Hermosa, Albquerque, Mexico).

14 American Institute of Aeronautics and Astronautics. Guide for the verification and validation of computational fluid dynamics simulations. American Institute of Aeronautics and Astronautics, G-0771998. 1998. 
15 Isaev, S. and Leont'ev, A. I. Analysis of the effect of viscosity on the vortex dynamics at laminar separated flow past a dimple on a plane with allowance for its asymmetry. J. Engng Phys. Thermophys., 2001, $74(2)$.

16 Mahmood, G. and Sabbagh, M. Z. Heat transfer in a channel with dimples and protrusions on opposite walls. J. Thermophys. Heat Transfer, 2001, 15(3), 275-283.

17 Burgess, N. and Olivera, M. M. Nusselt number behaviour on deep dimpled surface within a channel. J. Heat Transfer., 2003, 125, 11-18.

18 Chew, Y. and Khoo, B. C. Flow visualization studies on flow structures within spherical dimples of different depths with/without round edges. In Proceeding of 5th PSFVIP, PSFVIP-5-280, Australia, 27-29 September 2005, pp. 11.

19 Zerihan, J. and Zhang, J. Aerodynamics of a single element wing in ground effect. J. Aircraft, 2000, 37(6), 1058-1064.

20 Sovran, G. and Klomp, E. D. Experimentally determined optimum geometries for rectilinear diffusers with rectangular, canonical or annular cross-section. Fluids mechanics of internal flows, 1967, pp. 270-319 (Elsevier Publishing Co., New York).

21 Mittal, S. and Kumar, B. Flow past a rotating cylinder. J. Fluid Mech., 2003, 476, 303-334.

22 Fackrell, J. E. The aerodynamics of an isolated wheel in contact with the ground. Ph D Dissertation, Faculty of Engineering, University of London, 1974.

23 Fackrell, J. E. and Harvey, J. K. The aerodynamics of an isolated road wheel. In Proceedings of the Second Symposium on Aerodynamics of sports and competition automobiles, Los Angeles, California, USA, 1975, pp. 119-125, collection of AIAA papers (Western Periodicals Co., North Hollywood, California).

24 Axon, L. The aerodynamic characteristics of automobile wheels-CFD prediction and wind tunnel experiment. The aerodynamic characteristics of automobile wheels - CFD prediction and wind tunnel experiment.

25 Diasinos, S. The aerodynamic interaction of a rotating wheel and a downforce producing wing in ground effect. Ph D Dissertation, University of New South Wales, 2009.

26 Doig, G., Barber, T. J., Leonardi, E., Neely, A. J., and Kleine, H. Methods for investigating supersonic ground effect in a blowdown wind tunnel. Shock Waves, 2008, 18(2), 155-159.

27 Doig, G., Barber, T., Leonardi, E., and Neely, A. An investigation of flow field interactions with the ground around a supersonic land speed record vehicle using thermochromic liquid crystals and CFD. In Proceedings of the International Heat Transfer Conference, IHTC-13, Sydney, Australia, 13-18 August 2006, Paper EXP-19.

28 Aeschliman, D. and Oberkampf, W. Experimental methodology for computational fluid dynamics code validation. AIAA J., 1998, 36(5), 733-741.

29 Cook, P., McDonald, M. A., and Firmin, M. C. P. Aerofoil RAE 2822 -Pressure distributions, and boundary layer and wake measurements. Experimental data base for computer program assessment. AGARD Report AR 138, 1979.

30 Krige, D. A statistical approach to some basic mine valuation problems on the Witwatersrand. J. Chem. Metallurg. Mining Soc. South Africa, 1951, 52, 119-138.

31 Matheron, G. La thorie des variables rgionalises et ses applications, 1970 (Les Cahiers du Centre de Morphologie Mathmatique de Fontainebleau, Ecole Suprieure des Mines de Paris, Paris).

32 Laurenceau, J. and Sagaut, P. Building efficient response surfaces of aerodynamic functions with kriging and cokriging. AIAA J., 2008, 46(2), 498-507.

33 Wackernagel, H. Multivariate geostatistics: an introduction with applications, 2003 (Springer-Verlag, Berlin). 